\title{
Bayesian Estimation of Gumbel Type-II Distribution under Type-II Censoring with Medical Applications
}

\author{
Kamran Abbas $\mathbb{D}^{1},{ }^{1}$ Zamir Hussain, ${ }^{2}$ Noreen Rashid, ${ }^{3}$ Amjad Ali $\mathbb{D}^{4},{ }^{4}$ Muhammad Taj, ${ }^{5}$ \\ Sajjad Ahmad Khan, ${ }^{4}$ Sadaf Manzoor, ${ }^{4}$ Umair Khalil, ${ }^{6}$ and Dost Muhammad Khan $\mathbb{1}^{6}$ \\ ${ }^{1}$ Department of Statistics, University of Azad Jammu and Kashmir, Muzaffarabad, Pakistan \\ ${ }^{2}$ Research Centre for Modeling and Simulation, National University of Sciences and Technology, H-12 Campus, \\ Islamabad, Pakistan \\ ${ }^{3}$ Department of Statistics, Allama Iqbal Open University, Islamabad, Pakistan \\ ${ }^{4}$ Department of Statistics, Islamia College University, Peshawar, Khyber Pakhtunkhwa, Pakistan \\ ${ }^{5}$ Department of Mathematics, University of Azad Jammu and Kashmir, Muzaffarabad, Pakistan \\ ${ }^{6}$ Department of Statistics, Abdul Wali Khan University, Mardan, Khyber Pakhtunkhwa, Pakistan
}

Correspondence should be addressed to Kamran Abbas; kamiuajk@gmail.com

Received 11 December 2019; Revised 9 February 2020; Accepted 10 February 2020; Published 26 March 2020

Guest Editor: Pritee Khanna

Copyright $(92020$ Kamran Abbas et al. This is an open access article distributed under the Creative Commons Attribution License, which permits unrestricted use, distribution, and reproduction in any medium, provided the original work is properly cited.

The time to event or survival time usually follows certain skewed probability distributions. These distributions encounter vital role using the Bayesian framework to analyze and project the maximum life expectancy in order to inform decision-making. The Bayesian method provides a flexible framework for monitoring the randomized clinical trials to update what is already known using prior information about specific phenomena under uncertainty. Additionally, medical practitioners can use the Bayesian estimators to measure the probability of time until tumor recurrence, time until cardiovascular death, and time until AIDS for HIV patients by considering the prior information. However, in clinical trials and medical studies, censoring is present when an exact event occurrence time is not known. The present study aims to estimate the parameters of Gumbel type-II distribution based on the type-II censored data using the Bayesian framework. The Bayesian estimators cannot be obtained in explicit forms, and therefore we use Lindley's approximation based on noninformative prior and various loss functions such as squared error loss function, general entropy loss function, and LINEX (linear exponential) loss function. The maximum likelihood and Bayesian estimators are compared in terms of mean squared error by using the simulation study. Furthermore, two data sets about remission times (in months) of bladder cancer patients and survival times in weeks of 61 patients with inoperable adenocarcinoma of the lung are analyzed for illustration purposes.

\section{Introduction}

In medical research, data supporting the time until the occurrence of a particular event, such as the death of a patient, are frequently encountered. Such data are referred to as survival time data which has generally right-skewed distribution, and Gumbel type-II distribution can be used for this purpose. It was introduced by the German mathematician Gumbel in [1] and is useful to model "extreme values" such as floods, earthquakes, and natural disasters and also used in life expectancy tables, hydrology, and rainfall. The probability density function (PDF) of Gumbel type-II distribution is

$$
f(x \mid \alpha, \beta)=\alpha \beta x^{-(\alpha+1)} \exp \left(-\beta x^{-\alpha}\right), \quad x>0, \alpha, \beta>0,
$$

where " $\alpha$ " is the shape and " $\beta$ " is the scale parameter of the distribution. The corresponding cumulative distribution function $(\mathrm{CDF})$ is

$$
F(x \mid \alpha, \beta)=\exp \left(-\beta x^{-\alpha}\right) .
$$

A common feature of lifetime data is that the data points are possibly censored. In manifold reliability and life-testing 
studies, experiments are generally windup before failure times of all items are observed. Therefore, adequate information and results on failure times of all objects cannot be obtained. During experimentation, these situations occur due to loss or removal of objects before they fail. Therefore, generally, such experiments are preplanned and purposeful to save time and cost of these testing. Data obtained from such experiments are called censored. The type-I and type-II censoring are two well-known censoring schemes. In type-II censoring scheme, the number of failure units are fixed in advanced. For example, the investigator may decide to terminate the study after four of the six rats have developed tumors. There is an enormous literature accessible on estimation of parameters of distributions using type-II censoring, for example, Abbas and Tang [2] considered ML and least square estimators of Frechet distribution using type-II censored samples. Okasha [3] estimated the unknown parameters, reliability, and hazard functions of Lomax distribution under type-II censoring using Bayesian and E-Bayesian estimation. AbuZinadah [4] studied on exponentiated Gompertz distribution based on type-II and complete censored data. ElSagheer [5] studied the generalized pareto distribution under the different censoring schemes.

Recently, many authors have worked on Gumbel typeII distribution and Bayesian estimation using different loss functions. Abbas et al. [6] worked on Gumbel type-II distribution and obtained the Bayes estimators under different loss functions. Feroze and Aslam [7] obtained Bayes estimators of two components of Gumbel type-II distribution. Malinowska and Szynal [8] also derived Bayes estimators for Gumbel type-II distribution on kth lower record values. Sultana et al. [9] worked on a threecomponent mixture of Gumbel type-II distribution using Bayesian estimation under different priors such as informative and noninformative. Moreover, Metiri et al. [10] worked on the properties of the Lindley distribution. The Bayes estimates were derived under LINEX loss function using informative and noninformative priors (Reyad and Ahmed [11]). Preda et al. [12] developed Bayes estimators of modified Weibull distribution under squared error loss function (SELF) and LINEX loss function.

However, Bayesian estimation of Gumbel type-II distribution based on type-II censoring is not frequently discussed; therefore, we are interested in estimating the unknown parameters of Gumbel type-II distribution under type-II censored data. Including this introduction section, the rest of the paper is arranged as follows: in Section 2, maximum likelihood estimators (MLEs) for the parameters are obtained. In Section 3, Bayesian estimators based on different loss functions by taking noninformative and gamma priors are derived. The proposed estimators are compared in terms of their mean squared error (MSE) in Section 4. Section 5 illustrates the applications of proposed estimators with two examples, namely, data set of remission times for bladder cancer and survival times of inoperable adenocarcinoma of the lung. Finally, conclusions and recommendations are presented in Section 6.

\section{Maximum Likelihood Estimation}

Suppose that $X_{1}<X_{2}<, \ldots,<X_{r}$ is a type-II censored sample of size " $r$ " obtained from a life test on " $n$ " items whose life times have the Gumbel type-II distribution with parameters " $\alpha$ " and " $\beta$." The likelihood function of " $r$ " failures and $(n-r)$ censored values may be written as

$$
\begin{aligned}
& L=\left(\prod_{i=1}^{r} f\left(x_{i}, \theta\right)\right)\left(1-F\left(x_{r}, \theta\right)\right)^{n-r}, \\
& L=\left(\prod_{i=1}^{r} \alpha \beta x_{i}^{-(\alpha+1)} \exp \left(-\beta x_{i}^{-\alpha}\right)\right)\left(1-\exp \left(-\beta x_{r}^{-\alpha}\right)\right)^{n-r} .
\end{aligned}
$$

It is more convenient to work with log-likelihood. The log-likelihood function is

$$
\begin{aligned}
\ln L= & r \ln \alpha+r \ln \beta-(\alpha+1) \sum_{i=1}^{r} \ln x_{i}-\beta \sum_{i=1}^{r} x_{i}^{-\alpha} \\
& +(n-r) \ln \left(1-\exp \left(-\beta x_{r}^{-\alpha}\right)\right) .
\end{aligned}
$$

To get the ML estimator of $\alpha$ and $\beta$, differentiate equation (5) with respect to $\alpha$ and $\beta$ and the resulting equations are

$$
\begin{aligned}
\frac{\partial \ln L}{\partial \alpha}= & \frac{r}{\alpha}-\sum_{i=1}^{r} \ln x_{i}+\beta \sum_{i=1}^{r} x_{i}^{-\alpha} \ln x_{i}+(n-r) \\
& \cdot\left(\frac{\left(-\exp \left(-\beta x_{r}^{-\alpha}\right)\right)\left(\beta x_{r}^{-\alpha} \ln x_{r}\right)}{1-\exp \left(-\beta x_{r}^{-\alpha}\right)}\right)=0, \\
\frac{\partial \ln L}{\partial \beta}= & \frac{r}{\beta}-\sum_{i=1}^{r} x_{i}^{-\alpha}+(n-r) \\
& \cdot\left(\frac{-\exp \left(-\beta x_{r}^{-\alpha}\right)\left(-x_{r}^{-\alpha}\right)}{1-\exp \left(-\beta x_{r}^{-\alpha}\right)}\right)=0 .
\end{aligned}
$$

Equations (6) and (7) cannot be written in closed form. Therefore, here, we the use the Laplace approximation to get the point estimates of $\alpha$ and $\beta$.

\section{Bayesian Estimation}

In Bayesian estimation, we consider different loss functions such as squared error loss function (SELF) proposed by Legendre [13] and Gauss [14], LINEX (Varian [15]), and general entropy loss function (GELF) introduced by Calabria and Pulcini [16]. As both parameters are unknown, independent noninformative form of priors can be used. Supposed that $\alpha$ and $\beta$ have independent Gamma (a,b) and Gamma (c, d) priors, respectively, for $a, b, c, d>0$, i.e.,

$$
\begin{aligned}
& \pi_{1}(\alpha) \propto \alpha^{a-1}(\exp (-b \alpha)), \\
& \pi_{2}(\beta) \propto \beta^{c-1}(\exp (-d \beta)) .
\end{aligned}
$$

The joint prior distribution of parameters is 


$$
\begin{aligned}
\Phi^{\prime}(\alpha, \beta \mid x)= & K\left(\alpha^{(a+r-1)}\right)\left(\beta^{(c+r-1)}\right) \\
& \cdot\left(\exp \left(-b \alpha-d \beta-\beta \sum_{i=1}^{r} x_{i}^{-\alpha}\right)\right)\left(\prod_{i=1}^{r} x_{i}^{-\alpha-1}\right) \\
& \cdot\left(1-\exp \left(\sum_{i=1}^{r} x_{i}^{-\alpha}\right)\right)
\end{aligned}
$$

where $K$ is the normalizing constant that makes $\Phi^{\prime}(\alpha, \beta \mid x)$ a proper PDF. Thus,

$$
\begin{aligned}
K^{-1}= & \int_{\alpha, \beta}\left(\alpha^{(a+r-1)}\right)\left(\beta^{(c+r-1)}\right) \\
& \cdot\left(\exp \left(-b \alpha-d \beta-\beta \sum_{i=1}^{r} x_{i}^{-\alpha}\right)\right)\left(\prod_{i=1}^{r} x_{i}^{-\alpha-1}\right) \\
& \cdot\left(1-\exp \left(\sum_{i=1}^{r} x_{i}^{-\alpha}\right)\right) \mathrm{d} \alpha \mathrm{d} \beta .
\end{aligned}
$$

Therefore, the joint posterior density under any loss function is

$$
\Phi^{\prime}(\alpha, \beta \mid x)=\frac{\int_{\alpha} \int_{\beta} g(\alpha, \beta) L(\alpha, \beta) \pi_{1}(\alpha) \pi_{2}(\beta) \mathrm{d} \alpha \mathrm{d} \beta}{\int_{\alpha} \int_{\beta} L(\alpha, \beta) \pi_{1}(\alpha) \pi_{2}(\beta) \mathrm{d} \alpha \mathrm{d} \beta} .
$$

Posterior distribution (12) takes a ratio form that cannot be reduced to a closed form. Therefore, we use Lindley approximation [17] to get the Bayesian estimates, which can be written as

$$
\begin{aligned}
\widehat{g}= & g(\widehat{\alpha}, \widehat{\beta})+\frac{1}{2}\left[\sum_{i=1}^{2} \sum_{j=1}^{2} l_{i j} S_{i j}+l_{30} A_{12}+l_{03} A_{21}+l_{21} B_{12}+l_{12} B_{21}\right] \\
& +q_{1} C_{12}+q_{2} C_{21},
\end{aligned}
$$

where $\quad q_{1}=(\partial \ln \pi(\alpha, \beta) / \partial \alpha) ; \quad q_{2}=(\partial \ln \pi(\alpha, \beta) / \partial \beta)$; $l_{11}=\left(\partial^{2} g(\alpha, \beta) / \partial \alpha^{2}\right) ; \quad l_{12}=\left(\partial^{2} g(\alpha, \beta) / \partial \alpha \partial \beta\right) ; \quad l_{21}=$ $\left(\partial^{2} g(\alpha, \beta) / \partial \beta \partial \alpha\right) ; l_{22}=\left(\partial^{2} g(\alpha, \beta) / \partial \beta^{2}\right) ; l_{1}=(\partial g(\alpha, \beta) / \partial \alpha)$; $l_{2}=(\partial g(\alpha, \beta) / \partial \beta) ; \quad A_{i j}=\left(l_{i} S_{i i}+l_{j} S_{i j}\right) S_{i i} ; \quad B_{i j}=3 l_{i} S_{i i} S_{i j}+$ $l_{j}\left(S_{i i} S_{j j}+2 S_{i j}^{2}\right) ; C_{i j}=l_{i} S_{i i}+l_{j} S_{j i} i, j=1,2$. The detail of equation (13) is provided in Appendix. The approximate Bayesian estimators of " $\alpha$ " and " $\beta$ " based on SELF are

$$
\begin{aligned}
\widehat{\alpha}_{\mathrm{BSELF}}= & \widehat{\alpha}+\frac{1}{2}\left[\left\{\frac{2 r}{\hat{\alpha}^{3}}+\widehat{\beta} \sum_{i=1}^{r} x_{i}^{-\alpha}\left(\ln x_{i}\right)^{3}-\left(\frac{(n-r)(1-A) \widehat{\beta}\left(\ln x_{r}\right)^{3} x_{r}^{-\alpha}}{A^{3}}\right)\left(\widehat{\beta} x_{r}^{-\alpha}\left(\left(\hat{\beta} x_{r}^{-\alpha}-A\right)(2-A)-A(1+A)\right)+A^{2}\right)\right\}\right. \\
& S_{11}^{2}+\left\{\frac{2 r}{\hat{\beta}^{3}}-\frac{(n-r) x_{r}^{-3 \widehat{\alpha}}(1-A)(A-2)}{A^{3}}\right\} S_{21} S_{22} \\
& +3\left\{-\sum_{i=1}^{r} x_{i}^{-\widehat{\alpha}}\left(\ln x_{i}\right)^{2}-\left(\frac{(1-A)(n-r) x_{r}^{-\widehat{\alpha}}\left(\ln x_{r}\right)^{2}}{A^{3}}\right)\left(\widehat{\beta} x_{r}^{-\widehat{\alpha}}\left(\left(\widehat{\beta} x_{r}^{-\widehat{\alpha}}-A\right)(A-2)+A(A+1)\right)-A^{2}\right)\right\} \\
& \left.S_{11} S_{12}+\left\{-\frac{(n-r)(1-A) x_{r}^{-2 \widehat{\alpha}} \ln x_{r}}{A^{3}}\left(\widehat{\beta} x_{r}^{-\widehat{\alpha}}(2-A)-2 A\right)\right\}\left(S_{22} S_{21}+2 S_{21}^{2}\right)\right] \\
& +\left(\frac{a-1}{\widehat{\alpha}}-b\right) S_{11}+\left(\frac{c-1}{\widehat{\beta}}-d\right) S_{21},
\end{aligned}
$$




$$
\begin{aligned}
\widehat{\beta}_{\mathrm{BSELF}}= & \widehat{\beta}+\frac{1}{2}\left[\left\{\frac{2 r}{\hat{\alpha}^{3}}+\widehat{\beta} \sum_{i=1}^{r} x_{i}^{-\hat{\alpha}}\left(\ln x_{i}\right)^{3}-\left(\frac{(n-r)(1-A) \widehat{\beta}\left(\ln x_{r}\right)^{3} x_{r}^{\widehat{\alpha \alpha}}}{A^{3}}\right)\left(\widehat{\beta} x_{r}^{-\hat{\alpha}}\left(\left(\widehat{\beta} x_{r}^{-\widehat{\alpha}}-A\right)(2-A)-A(1+A)\right)+A^{2}\right)\right\}\right. \\
& S_{12} S_{11}+\left\{\frac{2 r}{\widehat{\beta}^{3}}-\frac{(n-r) x_{r}^{-3 \widehat{\alpha}}(1-A)(A-2)}{A^{3}}\right\} S_{22}^{2}+\left\{-\sum_{i=1}^{r} x_{i}^{-\widehat{\alpha}}\left(\ln x_{i}\right)^{2}-\left(\frac{(1-A)(n-r) x_{r}^{-\widehat{\alpha}}\left(\ln x_{r}\right)^{2}}{A^{3}}\right)\right. \\
& \left.\cdot\left(\widehat{\beta} x_{r}^{-\widehat{\alpha}}\left(\left(\widehat{\beta} x_{r}^{-\widehat{\alpha}}-A\right)(A-2)+A(A+1)\right)-A^{2}\right)\right\} \\
& \left.\left(S_{11} S_{22}+2 S_{12}^{2}\right)+3\left\{-\left(\frac{(n-r)(1-A) x_{r}^{-2 \widehat{\alpha}} \ln x_{r}}{A^{3}}\right)\left(\widehat{\beta} x_{r}^{-\widehat{\alpha}}(2-A)-2 A\right)\right\} S_{22} S_{21}\right] \\
& +\left(\frac{a-1}{\widehat{\alpha}}-b\right) S_{21}+\left(\frac{c-1}{\widehat{\beta}}-d\right) S_{22} .
\end{aligned}
$$

Similarly, the Bayesian estimators of " $\alpha$ " and " $\beta$ " under LINEX loss function are

$$
\begin{aligned}
& \widehat{\alpha}_{\text {BLINEX }}=-\frac{1}{k} \ln \left[e^{-\hat{\alpha}}+\frac{1}{2}\left\{k^{2} e^{-k \widehat{\alpha}} S_{11}-k e^{-k \widehat{\alpha}}\left(\frac{2 r}{\widehat{\alpha}^{3}}+\widehat{\beta} \sum_{i=1}^{r} x_{i}^{-\hat{\alpha}}\left(\ln x_{i}\right)^{3}-\left(\frac{(n-r)(1-A) \widehat{\beta}\left(\ln x_{r}\right)^{3} x_{r}^{-\hat{\alpha}}}{A^{3}}\right)\right.\right.\right. \\
& \left.\left(\widehat{\beta} x_{r}^{\hat{\alpha}}\left(\left(\widehat{\beta} x_{r}^{\hat{-\alpha}}-A\right)(2-A)-A(1+A)\right)+A^{2}\right)\right) S_{11}^{2} \\
& -k e^{-k \widehat{\alpha}}\left(\frac{2 r}{\hat{\beta}^{3}}-\frac{(n-r) x_{r}^{-3 \widehat{\alpha}}(1-A)(A-2)}{A^{3}}\right) S_{21} S_{22} \\
& -3 k e^{-\hat{\alpha}}\left(-\sum_{i=1}^{r} x_{i}^{-\widehat{\alpha}}\left(\ln x_{i}\right)^{2}-\left(\frac{(1-A)(n-r) x_{r}^{-\widehat{\alpha}}\left(\ln x_{r}\right)^{2}}{A^{3}}\right)\left(\widehat{\beta} x_{r}^{-\widehat{\alpha}}\left(\left(\widehat{\beta} x_{r}^{-\widehat{\alpha}}-A\right)(A-2)+A(A+1)\right)-A^{2}\right)\right) S_{11} S_{12} \\
& \left.-k e^{-\hat{k \alpha}}\left(-\left(\frac{(n-r)(1-A) x_{r}^{-2 \widehat{\alpha}} \ln x_{r}}{A^{3}}\right)\left(\widehat{\beta} x_{r}^{-\widehat{\alpha}}(2-A)-2 A\right)\right)\left(S_{22} S_{21}+2 S_{21}^{2}\right)\right\} \\
& \left.-k e^{-\hat{\alpha} \alpha}\left(\frac{a-1}{\widehat{\alpha}}-b\right) S_{11}-k e^{-\hat{k} \alpha}\left(\frac{c-1}{\widehat{\beta}}-d\right) S_{21}\right],
\end{aligned}
$$




$$
\begin{aligned}
& \widehat{\beta}_{\text {BLINEX }}=-\frac{1}{k} \ln \left[e^{-k \widehat{\beta}}+\frac{1}{2}\left\{k^{2} e^{-k \widehat{\beta}} S_{22}-k e^{-k \widehat{\beta}}\right.\right. \\
& \cdot\left(\frac{2 r}{\hat{\alpha}^{3}}+\widehat{\beta} \sum_{i=1}^{r} x_{i}^{\hat{-\alpha}}\left(\ln x_{i}\right)^{3}-\frac{(n-r)(1-A) \widehat{\beta}\left(\ln x_{r}\right)^{3} x_{r}^{\widehat{-\alpha}}}{A^{3}}\left(\widehat{\beta} x_{r}^{\widehat{-\alpha}}\left(\left(\widehat{\beta} x_{r}^{-\hat{\alpha}}-A\right)(2-A)-A(1+A)\right)+A^{2}\right)\right) \\
& S_{12} S_{11}-k e^{-k \hat{\beta}}\left(\frac{2 r}{\hat{\beta}^{3}}-\frac{(n-r) x_{r}^{-3 \widehat{\alpha}}(1-A)(A-2)}{A^{3}}\right) S_{22}^{2} \\
& -k e^{-\hat{k \beta}}\left(-\sum_{i=1}^{r} x_{i}^{-\widehat{\alpha}}\left(\ln x_{i}\right)^{2}-\frac{(1-A)(n-r) x_{r}^{-\widehat{\alpha}}\left(\ln x_{r}\right)^{2}}{A^{3}}\left(\widehat{\beta} x_{r}^{-\widehat{\alpha}}\left(\left(\widehat{\beta} x_{r}^{-\widehat{\alpha}}-A\right)(A-2)+A(A+1)\right)-A^{2}\right)\right) \\
& \cdot\left(S_{11} S_{22}+2 S_{12}^{2}\right) \\
& \left.-3 k e^{-k \hat{\beta}}\left(-\frac{(n-r)(1-A) x_{r}^{-2 \widehat{\alpha}} \ln x_{r}}{A^{3}}\left(\widehat{\beta} x_{r}^{-\widehat{\alpha}}(2-A)-2 A\right)\right) S_{22} S_{21}\right\} \\
& \left.-k e^{-k \hat{\beta}}\left(\frac{a-1}{\widehat{\alpha}}-b\right) S_{21}-k e^{-k \hat{\beta}}\left(\frac{c-1}{\widehat{\beta}}-d\right) S_{22}\right] .
\end{aligned}
$$

The Bayesian estimators of " $\alpha$ " and " $\beta$ " under GELF are

$$
\begin{aligned}
\widehat{\alpha}_{\mathrm{BGELF}}= & {\left[\widehat{\alpha}^{-k}+\frac{1}{2}\left\{k(k+1) \widehat{\alpha}^{-(k+2)} S_{11}-k \widehat{\alpha}^{-(k+1)}\right.\right.} \\
& \left(\frac{2 r}{\widehat{\alpha}^{3}}+\widehat{\beta} \sum_{i=1}^{r} x_{i}^{-\hat{\alpha}}\left(\ln x_{i}\right)^{3}-\frac{(n-r)(1-A) \hat{\beta}\left(\ln x_{r}\right)^{3} x_{r}^{-\widehat{\alpha}}}{A^{3}}\left(\beta x_{r}^{-\hat{\alpha}}\left(\left(\widehat{\beta} x_{r}^{-\hat{\alpha}}-A\right)(2-A)-A(1+A)\right)+A^{2}\right)\right) S_{11}^{2} \\
& -k \widehat{\alpha}^{-(k+1)}\left(\frac{2 r}{\widehat{\beta}^{3}}-\frac{(n-r) x_{r}^{-3 \widehat{\alpha}}(1-A)(A-2)}{A^{3}}\right) S_{21} S_{22} \\
& -3 k \widehat{\alpha}^{-(k+1)}-\left(\sum_{i=1}^{r} x_{i}^{-\hat{\alpha}}\left(\ln x_{i}\right)^{2}-\frac{(1-A)(n-r) x_{r}^{-\widehat{\alpha}}\left(\ln x_{r}\right)^{2}}{A^{3}}\left(\widehat{\beta} x_{r}^{-\widehat{\alpha}}\left(\left(\widehat{\beta} x_{r}^{-\widehat{\alpha}}-A\right)(A-2)+A(A+1)\right)-A^{2}\right)\right) S_{11} S_{12} \\
& \left.-k \widehat{\alpha}^{-(k+1)}\left(-\frac{(n-r)(1-A) x_{r}^{-2 \widehat{\alpha}} \ln x_{r}}{A^{3}}\left(\widehat{\beta} x_{r}^{-\widehat{\alpha}}(2-A)-2 A\right)\right)\left(S_{22} S_{21}+2 S_{21}^{2}\right)\right\} \\
& \left.-k \widehat{\alpha}^{-(k+1)}\left(\frac{a-1}{\widehat{\alpha}}-b\right) S_{11}-k \widehat{\alpha}^{-(k+1)}\left(\frac{c-1}{\widehat{\beta}}-d\right) S_{21}\right]^{-1 / k},
\end{aligned}
$$




$$
\begin{aligned}
\widehat{\beta}_{\mathrm{BGELF}}= & \widehat{\beta}^{-k}+\frac{1}{2}\left\{k(k+1) \widehat{\beta}^{-(k+2)} S_{22}\right. \\
& -k \widehat{\beta}^{-(k+1)}\left(\frac{2 r}{\hat{\alpha}^{3}}+\widehat{\beta} \sum_{i=1}^{r} x_{i}^{-\hat{\alpha}}\left(\ln x_{i}\right)^{3}-\frac{(n-r)(1-A) \widehat{\beta}\left(\ln x_{r}\right)^{3} x_{r}^{-\hat{\alpha}}}{A^{3}}\left(\widehat{\beta} x_{r}^{-\alpha}\left(\left(\widehat{\beta} x_{r}^{-\alpha}-A\right)(2-A)-A(1+A)\right)+A^{2}\right)\right) S_{12} S_{11} \\
& -k \widehat{\beta}^{-(k+1)}\left(\frac{2 r}{\widehat{\beta}^{3}}-\frac{(n-r) x_{r}^{-3 \widehat{\alpha}}(1-A)(A-2)}{A^{3}}\right) S_{22}^{2}-k \widehat{\beta}^{-(k+1)} \\
& \cdot\left(-\sum_{i=1}^{r} x_{i}^{-\hat{\alpha}}\left(\ln x_{i}\right)^{2}-\frac{(1-A)(n-r) x_{r}^{-\widehat{\alpha}}\left(\ln x_{r}\right)^{2}}{A^{3}}\left(\widehat{\beta} x_{r}^{-\hat{\alpha}}\left(\left(\widehat{\beta} x_{r}^{-\widehat{\alpha}}-A\right)(A-2)+A(A+1)\right)-A^{2}\right)\right)\left(S_{11} S_{22}+2 S_{12}^{2}\right) \\
& -3 k \widehat{\beta}^{-(k+1)}\left(-\frac{\left.\left.(n-r)(1-A) x_{r}^{-2 \widehat{\alpha}} \ln x_{r}\left(\widehat{\beta} x_{r}^{-\widehat{\alpha}}(2-A)-2 A\right)\right) S_{22} S_{21}\right\}}{A^{3}}\right. \\
& \left.-k \widehat{\beta}^{-(k+1)}\left(\frac{a-1}{\widehat{\alpha}}-b\right) S_{21}-k \widehat{\beta}^{-(k+1)}\left(\frac{c-1}{\widehat{\beta}}-d\right) S_{22}\right]^{-1 / k},
\end{aligned}
$$

where $\widehat{\alpha}$ and $\widehat{\beta}$ are the ML estimators of $\alpha$ and $\beta$ which can be obtained from equations (6) and (7), respectively.

\section{Simulation Study}

The performance of the proposed Bayesian estimators with their ML counterpart in terms of MSE, different sample sizes, and different values of parameters are considered using Monte Carlo simulation based on prespecified different percentages of failures, i.e., $40 \%, 60 \%$, and $80 \%$. Monte Carlo simulation is conducted as follows:

(i) Take the initial values of $\alpha$ and $\beta$, respectively, and the samples are generated from the Gumbel type-II distribution using inverse transformation technique, i.e., $X(F)=(-(\log U / \beta))^{-(1 / \alpha)}$, where $U \sim$ uniform $(0,1)$.

(ii) First, calculate the MLE using Laplace approximation, and then Bayesian estimates under noninformative priors are obtained via Lindley approximation.

(iii) The process is replicated 5000 times for each sample size and averages of these estimates and the corresponding MSEs (within parenthesis) were calculated for each method using the $\mathrm{R}$ software version (i386 3.6.1), which approximately takes around half an hour.

The results are reported in Tables $1-4$ for comparison purposes. Tables 1 and 2 contain simulation results for the case where loss function parameter $k=1$ and values of hyperparameters are considered as $a=b=c=d=2$, whereas Tables 3 and 4 comprise the results for the case where $k=1.5$ and values of hyperparameters are $a=1, b=2, c=2.25$, and $d=1.5$ for the simulation study. From the results of the simulation study, conclusions are drawn regarding the behavior of the estimators, which are summarized as follows:

(i) In terms of MSEs, the ML and Bayesian estimators become closer by increasing the sample sizes. (ii) For fixed percentage of failures, as sample size increases, it is observed that the MSEs of all the estimators decrease because as for large sample sizes, prior has minimal effect on the posterior.

(iii) For fixed values of $\alpha$ and $\beta$, the MSEs of ML and Bayesian estimators decrease when both increase the sample size and percentage of failures.

(iv) When $k=1$ and $a=b=c=d=2$, the Bayesian estimators based on GELF and LINEX loss function are smaller as compared to ML estimators in terms of MSEs. Therefore, Bayes estimators are much stable than ML estimators.

(v) Generally, the ML and Bayesian estimators are closed for the large sample in terms of MSE.

\section{Data Analysis}

In this section, we consider two examples for illustration purposes.

5.1. Example 1. The real data about remission times (in months) of a random sample of 128 bladder cancer patients presented in Table 5 were reported by Lee and Wang [20]. A total of 128 patients with different prespecified percentages of events, i.e., $40 \%, 50 \%, 60 \%$, and $80 \%$, represented patients whose treatment was terminated and rest of the percentages are censored. Clearly, Figure 1 confirms that the histogram is slightly skewed to the right and is leptokurtic. Moreover, ML and Bayesian estimates can also be envisioned in Figure 1, in which the $x$-axis represents the remission times (in months) of bladder cancer patients, while the Gumbel type-II density function is taken on the $y$ axis. Therefore, it would be appropriate to select positively skewed distributions for describing the behavior of remission times of bladder cancer patients. Amongst the skewed distributions, Gumbel type-II distribution is fitted and the parameter estimates using ML and Bayesian methods are presented in Table 6 for comparison purposes. 
TABLE 1: Average ML and Bayesain estimates with corresponding MSEs (within parenthesis) using different percentages of failures for $\alpha=1.5$ (when $a=b=c=d=2, k=1$ ).

\begin{tabular}{|c|c|c|c|c|c|}
\hline$n$ & Percent & ML & BSELF & BGELF & BLINEX \\
\hline \multirow{3}{*}{25} & 40 & $1.7162(0.2808)$ & $1.7948(0.3524)$ & $1.4560(0.0989)$ & $1.4666(0.0981)$ \\
\hline & 60 & $1.6408(0.1565)$ & $1.6893(0.1822)$ & $1.4633(0.0794)$ & $1.4708(0.0758)$ \\
\hline & 80 & $1.6160(0.1090)$ & $1.6527(0.1231)$ & $1.4883(0.0645)$ & $1.4947(0.0620)$ \\
\hline \multirow{3}{*}{50} & 40 & $1.6263(0.0985)$ & $1.6580(0.1112)$ & $1.5114(0.0587)$ & $1.5179(0.0565)$ \\
\hline & 60 & $1.5610(0.0579)$ & $1.5826(0.0624)$ & $1.4836(0.0429)$ & $1.4891(0.0416)$ \\
\hline & 80 & $1.5667(0.0412)$ & $1.5840(0.0446)$ & $1.5084(0.0308)$ & $1.5126(0.0303)$ \\
\hline \multirow{3}{*}{80} & 40 & $1.5708(0.0521)$ & $1.5889(0.0561)$ & $1.5042(0.0384)$ & $1.5095(0.0375)$ \\
\hline & 60 & $1.5516(0.0348)$ & $1.5648(0.0369)$ & $1.5041(0.0278)$ & $1.5080(0.0274)$ \\
\hline & 80 & $1.5269(0.0223)$ & $1.5373(0.0233)$ & $1.4926(0.0196)$ & $1.4958(0.0193)$ \\
\hline \multirow{3}{*}{100} & 40 & $1.5540(0.0420)$ & $1.5681(0.0445)$ & $1.5023(0.0334)$ & $1.5069(0.0328)$ \\
\hline & 60 & $1.5345(0.0278)$ & $1.5448(0.0289)$ & $1.4975(0.0237)$ & $1.5008(0.0234)$ \\
\hline & 80 & $1.5292(0.0176)$ & $1.5374(0.0184)$ & $1.5020(0.0155)$ & $1.5045(0.0154)$ \\
\hline \multirow{3}{*}{150} & 40 & $1.5327(0.0243)$ & $1.5448(0.0253)$ & $1.4992(0.0210)$ & $1.5026(0.0207)$ \\
\hline & 60 & $1.5234(0.0162)$ & $1.5381(0.0167)$ & $1.4991(0.0146)$ & $1.5016(0.0145)$ \\
\hline & 80 & $1.5143(0.0128)$ & $1.5208(0.0131)$ & $1.4977(0.0119)$ & $1.4995(0.0118)$ \\
\hline \multirow{3}{*}{200} & 40 & $1.5317(0.0188)$ & $1.5384(0.0195)$ & $1.5067(0.0165)$ & $1.5092(0.0164)$ \\
\hline & 60 & $1.5220(0.0114)$ & $1.5271(0.0117)$ & $1.5040(0.0104)$ & $1.5058(0.0103)$ \\
\hline & 80 & $1.5119(0.0089)$ & $1.5160(0.0091)$ & $1.4987(0.0084)$ & $1.5001(0.0083)$ \\
\hline
\end{tabular}

ML: maximum likelihood estimators, BSELF: Bayesian estimators under squared error loss function, BGELF: Bayesian estimators under general entropy loss function, and BLINEX: Bayesian estimators under linear exponential loss function.

TABle 2: Average ML and Bayesian estimates with corresponding MSEs (within parenthesis) using different percentages of failures for $\beta=1.5$ (when $a=b=c=d=2, k=1$ ).

\begin{tabular}{|c|c|c|c|c|c|}
\hline$n$ & Percent & ML & BSELF & BGELF & BLINEX \\
\hline \multirow{3}{*}{25} & 40 & $1.5418(0.1538)$ & $1.6030(0.1852)$ & $1.3879(0.1131)$ & $1.3988(0.1042)$ \\
\hline & 60 & $1.5907(0.1522)$ & $1.6488(0.1737)$ & $1.4357(0.0886)$ & $1.4448(0.0842)$ \\
\hline & 80 & $1.5725(0.1338)$ & $1.6280(0.1651)$ & $1.4255(0.0827)$ & $1.4346(0.0782)$ \\
\hline \multirow{3}{*}{50} & 40 & $1.5211(0.0635)$ & $1.5473(0.0684)$ & $1.4455(0.0548)$ & $1.4521(0.0526)$ \\
\hline & 60 & $1.5396(0.0575)$ & $1.5644(0.0632)$ & $1.4677(0.0467)$ & $1.4735(0.0451)$ \\
\hline & 80 & $1.5358(0.0565)$ & $1.5609(0.0624)$ & $1.4658(0.0458)$ & $1.4715(0.0442)$ \\
\hline \multirow{3}{*}{80} & 40 & $1.5147(0.0351)$ & $1.5303(0.0371)$ & $1.4676(0.0320)$ & $1.4721(0.0312)$ \\
\hline & 60 & $1.5215(0.0349)$ & $1.5364(0.0364)$ & $1.4774(0.0311)$ & $1.4814(0.0304)$ \\
\hline & 80 & $1.5262(0.0341)$ & $1.5415(0.0363)$ & $1.4830(0.0297)$ & $1.4869(0.0291)$ \\
\hline \multirow{3}{*}{100} & 40 & $1.5026(0.0298)$ & $1.5149(0.0306)$ & $1.4654(0.0285)$ & $1.4691(0.0279)$ \\
\hline & 60 & $1.5220(0.0260)$ & $1.5338(0.0272)$ & $1.4868(0.0237)$ & $1.4901(0.0233)$ \\
\hline & 80 & $1.5158(0.0249)$ & $1.5278(0.0262)$ & $1.4817(0.0224)$ & $1.4850(0.0221)$ \\
\hline \multirow{3}{*}{150} & 40 & $1.5033(0.0191)$ & $1.5114(0.0194)$ & $1.4784(0.0184)$ & $1.4809(0.0182)$ \\
\hline & 60 & $1.5118(0.0165)$ & $1.5194(0.0170)$ & $1.4886(0.0157)$ & $1.4909(0.0155)$ \\
\hline & 80 & $1.5067(0.0163)$ & $1.5145(0.0168)$ & $1.4842(0.0153)$ & $1.4865(0.0151)$ \\
\hline \multirow{3}{*}{200} & 40 & $1.5060(0.0130)$ & $1.5121(0.0133)$ & $1.4873(0.0123)$ & $1.4892(0.0122)$ \\
\hline & 60 & $1.5105(0.0119)$ & $1.5162(0.0122)$ & $1.4931(0.0113)$ & $1.4949(0.0112)$ \\
\hline & 80 & $1.5110(0.0116)$ & $1.5169(0.0118)$ & $1.4940(0.0112)$ & $1.4958(0.0111)$ \\
\hline
\end{tabular}

TABle 3: Average ML and Bayesian estimates with corresponding MSEs (within parenthesis) using different percentages of failures for $\alpha=1.5$ (when $a=1, b=2, c=2.25, d=1.5$, and $k=1.5$ ).

\begin{tabular}{|c|c|c|c|c|c|}
\hline$n$ & Percent & ML & BSELF & BGELF & BLINEX \\
\hline \multirow{3}{*}{50} & 40 & $1.5879(0.0923)$ & $1.6185(0.1025)$ & $1.4239(0.0629)$ & $1.4251(0.0605)$ \\
\hline & 60 & $1.5757(0.0594)$ & $1.5975(0.0649)$ & $1.4604(0.0422)$ & $1.4615(0.0407)$ \\
\hline & 80 & $1.5404(0.0451)$ & $1.5575(0.0477)$ & $1.4576(0.0374)$ & $1.4590(0.0363)$ \\
\hline \multirow{3}{*}{80} & 40 & $1.5434(0.0408)$ & $1.5607(0.0435)$ & $1.4439(0.0338)$ & $1.4454(0.0326)$ \\
\hline & 60 & $1.5704(0.0382)$ & $1.5838(0.0408)$ & $1.4973(0.0296)$ & $1.4983(0.0270)$ \\
\hline & 80 & $1.5493(0.0200)$ & $1.5597(0.0214)$ & $1.4479(0.0278)$ & $1.4982(0.0151)$ \\
\hline \multirow{3}{*}{100} & 40 & $1.5280(0.0337)$ & $1.5419(0.0354)$ & $1.4599(0.0171)$ & $1.4495(0.0288)$ \\
\hline & 60 & $1.5145(0.0179)$ & $1.5247(0.0202)$ & $1.4973(0.0161)$ & $1.4612(0.0167)$ \\
\hline & 80 & $1.5389(0.0173)$ & $1.5472(0.0186)$ & $1.4971(0.0154)$ & $1.4982(0.0150)$ \\
\hline \multirow{3}{*}{200} & 40 & $1.5370(0.0158)$ & $1.5439(0.0165)$ & $1.4959(0.0131)$ & $1.4968(0.0129)$ \\
\hline & 60 & $1.5184(0.0114)$ & $1.5248(0.0117)$ & $1.4903(0.0104)$ & $1.4910(0.0103)$ \\
\hline & 80 & $1.5216(0.0113)$ & $1.5257(0.0116)$ & $1.5007(0.0103)$ & $1.5013(0.0103)$ \\
\hline
\end{tabular}


TABLE 4: Average ML and Bayesian estimates with corresponding MSEs (within parenthesis) using different percentages of failures for $\beta=1.5$ (when $a=1, b=2, c=2.25, d=1.5$, and $k=1.5$ ).

\begin{tabular}{|c|c|c|c|c|c|}
\hline$n$ & Percent & ML & BSELF & BGELF & BLINEX \\
\hline \multirow{3}{*}{50} & 40 & $1.5341(0.0661)$ & $1.5818(0.0734)$ & $1.5067(0.0546)$ & $1.5085(0.0521)$ \\
\hline & 60 & $1.5564(0.0627)$ & $1.5719(0.0693)$ & $1.4987(0.0524)$ & $1.5006(0.0501)$ \\
\hline & 80 & $1.5464(0.0494)$ & $1.5599(0.0536)$ & $1.4829(0.0430)$ & $1.4856(0.0408)$ \\
\hline \multirow{3}{*}{80} & 40 & $1.5052(0.0382)$ & $1.5459(0.0407)$ & $1.5008(0.0342)$ & $1.5024(0.0332)$ \\
\hline & 60 & $1.5308(0.0319)$ & $1.5208(0.0330)$ & $1.4741(0.0302)$ & $1.4763(0.0292)$ \\
\hline & 80 & $1.5187(0.0285)$ & $1.5337(0.0301)$ & $1.4901(0.0260)$ & $1.4919(0.0254)$ \\
\hline \multirow{3}{*}{100} & 40 & $1.5209(0.0261)$ & $1.5333(0.0272)$ & $1.4954(0.0241)$ & $1.4970(0.0235)$ \\
\hline & 60 & $1.5079(0.0254)$ & $1.5195(0.0263)$ & $1.4846(0.0240)$ & $1.4862(0.0234)$ \\
\hline & 80 & $1.5075(0.0212)$ & $1.5193(0.0221)$ & $1.4851(0.0200)$ & $1.4867(0.0195)$ \\
\hline \multirow{3}{*}{200} & 40 & $1.5210(0.0151)$ & $1.5269(0.0156)$ & $1.5194(0.0148)$ & $1.4794(0.0146)$ \\
\hline & 60 & $1.5310(0.0149)$ & $1.5371(0.0155)$ & $1.5092(0.0144)$ & $1.5099(0.0142)$ \\
\hline & 80 & $1.4907(0.0147)$ & $1.4967(0.0149)$ & $1.4783(0.0138)$ & $1.5201(0.0137)$ \\
\hline
\end{tabular}

TABle 5: Remission times (in months) of a random sample of 128 bladder cancer patients.

$0.08,2.09,3.48,4.87,6.94,8.66,13.11,23.63,0.20,2.23,3.52,4.98$ $6.97,9.02,13.29,0.40,2.26,3.57,5.06,7.09,9.22,13.80,25.74,0.50$ $2.46,3.64,5.09,7.26,9.47,14.24,25.82,0.51,2.54,3.70,5.17,7.28$ $9.74,14.76,6.31,0.81,2.62,3.82,5.32,7.32,10.06,14.77,32.15,2.64$ $3.88,5.32,7.39,10.34,14.83,34.26,0.90,2.69,4.18,5.34,7.59$, $10.66,15.96,36.66,1.05,2.69,4.23,5.41,7.62,10.75,16.62,43.01$, $1.19,2.75,4.26,5.41,7.63,17.12,46.12,1.26,2.83,4.33,5.49,7.66$, $11.25,17.14,79.05,1.35,2.87,5.62,7.87,11.64,17.36,1.40,3.02$, $4.34,5.71,7.93,11.79,18.10,1.46,4.40,5.85,8.26,11.98,19.13$, $1.76,3.25,4.50,6.25,8.37,12.02,2.02,3.31,4.51,6.54,8.53,12.03$, $20.28,2.02,3.36,6.76,12.07,21.73,2.07,3.36,6.93,8.65,12.63$, 22.69

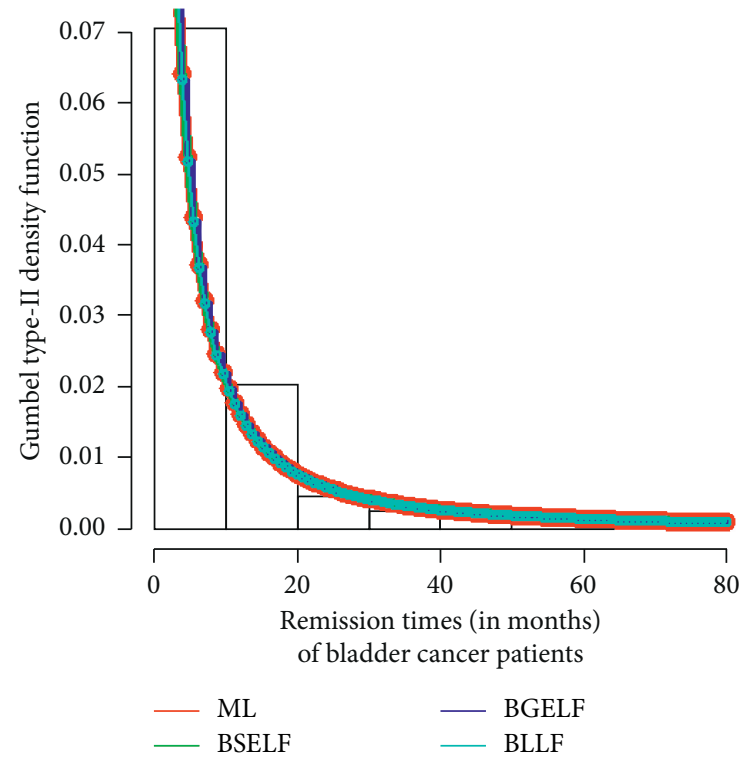

Figure 1: Comparison of estimation methods.

It is concluded that the proposed estimators of Gumbel type-II distribution fit the data well. Therefore, it is recommended that the Bayesian estimators can be more
TABle 6: Point estimates of $\alpha$ and $\beta$ using different percentages of failures when $a=1, b=2, c=2.25, d=1.5$, and $k=1$.

\begin{tabular}{cccccc}
\hline & Percent & ML & BSELF & BGELF & BLINEX \\
\hline \multirow{4}{*}{$\alpha$} & 40 & 0.5433 & 0.5452 & 0.5287 & 0.5329 \\
& 50 & 0.5909 & 0.5921 & 0.5779 & 0.5812 \\
& 60 & 0.6339 & 0.6348 & 0.6225 & 0.6252 \\
& 80 & 0.6988 & 0.6994 & 0.6898 & 0.6917 \\
\hline \multirow{4}{*}{$\beta$} & 40 & 2.4491 & 2.4702 & 2.3960 & 2.3915 \\
& 50 & 2.4252 & 2.4440 & 2.3729 & 2.3689 \\
& 60 & 2.4139 & 2.4312 & 2.3623 & 2.3594 \\
& 80 & 2.4095 & 2.4253 & 2.3588 & 2.3551 \\
\hline
\end{tabular}

TABLE 7: Survival times in weeks of 61 patients with inoperable adenocarcinoma of the lung.

\begin{tabular}{l}
\hline 28 Censored observations \\
\hline $0.14,0.14,0.29,0.43,0.57,0.57,1.86,3.00,3.00,3.29,3.29,6.00$ \\
$6.00,6.14,8.71,10.57,11.86,15.57,16.57,17.29,18.71,21.29,23.86$, \\
$26.00,27.57,32.14,33.14,47.29$ \\
\hline 33 Uncensored observations \\
\hline $0.43,2.86,3.14,3.14,3.43,3.43,3.71,3.86,6.14,6.86,9.00,9.43$ \\
$10.71,10.86,11.14,13.00,14.43,15.71,18.43,18.57,20.71,29.14$, \\
$29.71,40.57,48.57,49.43,53.86,61.86,66.57,68.71,68.96,72.86$, \\
72.86
\end{tabular}

beneficial to address the uncertainty in medical-related censored data.

5.2. Example 2. The survival times, in weeks, of 61 patients with unoperable lung cancer treated with cyclophosphamide considered in Lagakos and Williams ([18]) and in Lee and Wolfe ([19]) are presented in Table 7. There are 33 uncensored observations and 28 censored observations, representing the patients whose treatment was terminated because of a devolving condition. The point estimates of $\alpha$ and $\beta$ obtained by all the methods are summarized in Table 8. Figure 2 shows the results of different estimation methods and depicts that Gumbel type-II distribution fits the data better, in which $x$-axis comprises the survival times in weeks of 61 patients with inoperable adenocarcinoma of 
TABLe 8: Point estimates of $\alpha$ and $\beta$ when $a=1, b=2, c=2.25$, $d=1.5$, and $k=1$.

\begin{tabular}{lcccc}
\hline Parameter & ML & BSELF & BGELF & BLINEX \\
\hline$\alpha$ & 0.4354817 & 0.4398853 & 0.4218823 & 0.4277962 \\
$\beta$ & 2.121879 & 2.156254 & 2.049693 & 2.047704 \\
\hline
\end{tabular}

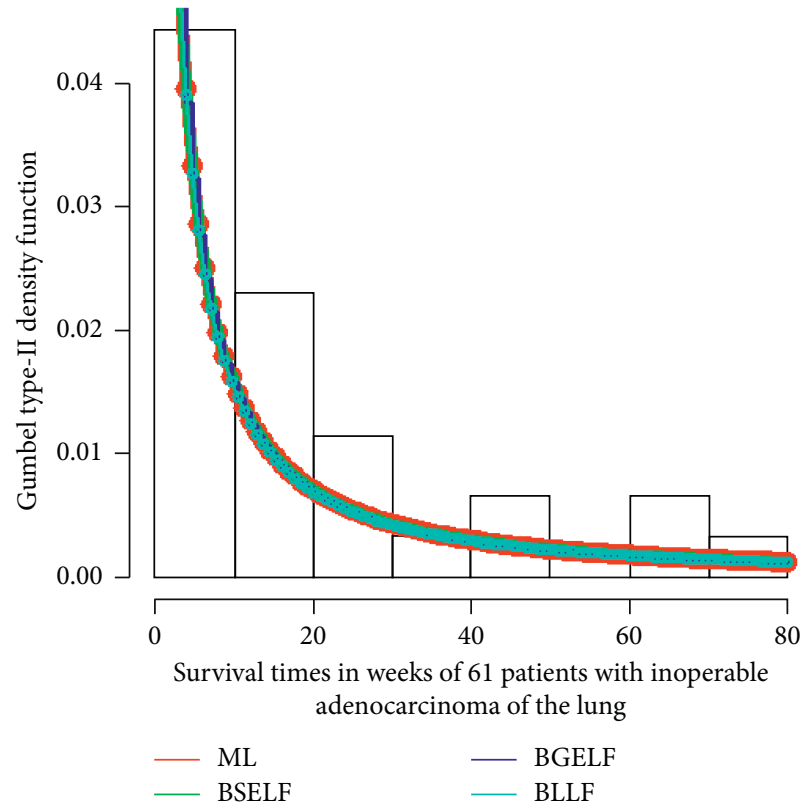

Figure 2: Comparison of estimation methods.

the lung as the Gumbel type-II density function is taken on the $y$-axis.

\section{Conclusion and Recommendations}

In medical decision-making, Bayesian tools incorporate the state of uncertainty and provide a rational framework for studying such problems. Usually, medical data are generally skewed to the right, and positively skewed distributions can be most suitable for describing unimodal medical data. In this study, an attempt has been made to develop the Bayesian estimators for Gumbel type-II distribution based on type-II censored data using squared error loss, GELF, and LINEX loss functions via Lindley's approximation. It is concluded that ML and Bayesian estimators become closer by increasing the sample sizes and prespecified percentages of failures. Based on the outcomes of this research study, we may suggest that this study can be further extended by using other skewed distributions considering the Bayesian framework with other loss functions using medical data.

\section{Appendix}

\section{Observed Fisher Information Matrix}

The observed Fisher information matrix (FIM) is computed by taking the $2^{\text {nd }}$ partial derivatives with respect to " $\alpha$ " and " $\beta$," respectively. Therefore, the matrix may be defined as

$$
I_{(\widehat{\alpha}, \widehat{\beta})}=\left[\begin{array}{cc}
-\frac{\partial^{2} \ln L}{\partial \alpha^{2}} & -\frac{\partial^{2} \ln L}{\partial \alpha \partial \beta} \\
-\frac{\partial^{2} \ln L}{\partial \beta \partial \alpha} & -\frac{\partial^{2} \ln L}{\partial \beta^{2}}
\end{array}\right] .
$$

The components of observed FIM are

$$
\begin{aligned}
\frac{\partial^{2} \ln L}{\partial \alpha^{2}}= & -\frac{r}{\alpha^{2}}-\beta \sum_{i=1}^{r} x_{i}^{-\alpha}\left(\ln x_{i}\right)^{2}-(n-r) \beta \ln x_{r} \\
& \cdot(1-A) x_{r}^{-\alpha} \ln x_{r}\left[\frac{x_{r}^{-\alpha} \beta-A}{A^{2}}\right], \\
& \cdot \frac{r}{\alpha^{2}}+\beta \sum_{i=1}^{r} x_{i}^{-\alpha}\left(\ln x_{i}\right)^{2}+(n-r) \beta \ln x_{r} \\
& \cdot(1-A) x_{r}^{-\alpha} \ln x_{r}\left[\frac{x_{r}^{-\alpha} \beta-A}{A^{2}}\right]=U, \\
\frac{\partial^{2} \ln L}{\partial \beta^{2}}= & -\frac{r}{\beta^{2}}-(n-r) x_{r}^{-2 \alpha}\left[\frac{(1-A)}{A^{2}}\right], \\
& \cdot \frac{r}{\beta^{2}}+(n-r) x_{r}^{-2 \alpha}\left[\frac{(1-A)}{A^{2}}\right]=V, \\
\frac{\partial^{2} \ln L}{\partial \beta \partial \alpha}=\sum_{i=1}^{r} & x_{i}^{-\alpha} \ln x_{i}+(n-r)\left[\frac{(1-A) x_{r}^{-\alpha} \ln x_{r}\left(\beta x_{r}^{-\alpha}-A\right)}{A^{2}}\right], \\
& -\sum_{i=1}^{r} x_{i}^{-\alpha} \ln x_{i}-(n-r) \\
& \cdot\left[\frac{(1-A) x_{r}^{-\alpha} \ln x_{r}\left(\beta x_{r}^{-\alpha}-A\right)}{A^{2}}\right]=W .
\end{aligned}
$$

The observed FIM matrix is rewritten as

$$
I_{(\alpha, \beta)}=\left[\begin{array}{cc}
U & W \\
W & V
\end{array}\right] .
$$

The inverse of $I_{(\alpha, \beta)}$ is

$$
\begin{aligned}
I_{(\alpha, \beta)}^{-1} & =\left[\begin{array}{ll}
S_{11} & S_{12} \\
S_{21} & S_{22}
\end{array}\right], \\
S_{11} & =\frac{V}{U V-W^{2}}, \\
S_{12} & =-\frac{W}{U V-W^{2}}, \\
S_{21} & =-\frac{W}{U V-W^{2}}, \\
S_{22} & =\frac{U}{U V-W^{2}},
\end{aligned}
$$


where $l_{i j}=\left(\partial^{i+j}\left(\alpha_{1}, \alpha_{2}\right) / \partial \alpha_{1}^{i} \alpha_{2}^{i}\right) ; i, j=0,1,2,3 . q_{1}=(\partial \ln$ $\pi(\alpha, \beta) / \partial \alpha), q_{2}=(\partial \ln \pi(\alpha, \beta) / \partial \beta), l_{11}=\left(\partial^{2} g(\alpha, \beta) / \partial \alpha^{2}\right), l_{12}=$ $\left(\partial^{2} g(\alpha, \beta) / \partial \alpha \partial \beta\right), l_{21}=\left(\partial^{2} g(\alpha, \beta) / \partial \beta \partial \alpha\right), l_{22}=\left(\partial^{2} g(\alpha, \beta)\right.$ $\left./ \partial \beta^{2}\right), l_{1}=(\partial g(\alpha, \beta) / \partial \alpha) ; l_{2}=(\partial g(\alpha, \beta) / \partial \beta), A_{i j}=\left(l_{i} S_{i i}+l_{j} S_{i j}\right)$ $\left.S_{i i}\right), B_{i j}=3 l_{i} S_{i i} S_{i j}+l_{j}\left(S_{i i} S_{j j}+2 S_{i j}^{2}\right)$, and $C_{i j}=l_{i} S_{i i}+l_{j} S_{j i}, i, j=1,2$.

$$
\begin{aligned}
\sum_{i=1}^{2} \sum_{i=1}^{2} l_{i j} S_{i j} & =l_{11} S_{11}+l_{12} S_{12}+l_{21} S_{21}+l_{22} S_{22}, \\
l_{1} & =1, l_{2}=l_{11}=l_{12}=l_{21}=l_{22}=0 \\
\sum_{i=1}^{2} \sum_{i=1}^{2} l_{i j} S_{i j} & =0
\end{aligned}
$$

$A_{i j}=\left(l_{i} S_{i i}+l_{j} S_{i j}\right) S_{i i}, A_{12}=\left(l_{1} S_{11}+l_{2} S_{12}\right) S_{11}, A_{12}=S_{11}^{2}, A_{21}=$ $\left(l_{2} S_{22}+l_{1} S_{21}\right) S_{22}, A_{21}=S_{21} S_{22}, B_{i j}=3 l_{i} S_{i i} S_{i j}+l_{j}\left(S_{i i} S_{j j}+2 S_{i j}^{2}\right)$, $B_{12}=3 l_{1} S_{11} S_{12}+l_{2}\left(S_{11} S_{22}+2 S_{12}^{2}\right), \quad B_{12}=3 S_{11} S_{12}, \quad B_{21}=$ $3 l_{2} S_{22} S_{21}+l_{1}\left(S_{22} S_{11}+2 S_{21}^{2}\right), \quad B_{21}=\left(S_{22} S_{11}+2 S_{21}^{2}\right), \quad C_{i j}=$ $l_{i} S_{i i}+l_{j} S_{j i}, \quad C_{12}=l_{1} S_{11}+l_{2} S_{21}, \quad C_{12}=S_{11}, \quad C_{21}=l_{2} S_{22}+l_{1} S_{12}$, $C_{21}=S_{12}, q_{1}=(((a-1) / \widehat{\alpha})-b), q_{2}=(((c-1) / \widehat{\beta})-d)$,

$$
\begin{aligned}
\frac{\partial^{3} \ln L}{\partial \alpha^{3}}= & \frac{2 r}{\alpha^{3}}+\beta \sum_{i=1}^{r} x_{i}^{-\alpha}\left(\ln x_{i}\right)^{3}-(n-r) \beta\left(\ln x_{r}\right)^{2}\left[\frac{(1-A)}{A^{2}} x_{r}^{-\alpha}\left(-\beta x_{r}^{-\alpha} \ln x_{r}+\exp \left(-\beta x_{r}^{-\alpha}\right)\left(\beta x_{r}^{-\alpha} \ln x_{r}\right)\right)\right. \\
& +\frac{(1-A)}{A^{2}}\left(\beta x_{r}^{-\alpha}-A\right)\left(-x_{r}^{-\alpha} \ln x_{r}\right) \\
& \left.+\frac{x_{r}^{-\alpha}\left(\beta x_{r}^{-\alpha}-A\right)}{\left(1-\exp \left(-\beta x_{r}^{-\alpha}\right)\right)^{4}}\left(\left(1-\exp \left(-\beta x_{r}^{-\alpha}\right)\right)^{2} \exp \left(-\beta x_{r}^{-\alpha}\right) \beta x_{r}^{-\alpha} \ln x_{r}+2\left(\exp \left(-\beta x_{r}^{-\alpha}\right)\right)^{2}\left(1-\exp \left(-\beta x_{r}^{-\alpha}\right)\right) \beta x_{r}^{-\alpha} \ln x_{r}\right)\right],
\end{aligned}
$$

$$
\begin{aligned}
\frac{\partial^{3} \ln L}{\partial \beta^{3}}= & \frac{2 r}{\beta^{3}}-\frac{(n-r) x_{r}^{-2 \alpha}}{\left(1-\exp \left(-\beta x_{r}^{-\alpha}\right)\right)^{4}} \\
& {\left[\left(1-\exp \left(-\beta x_{r}^{-\alpha}\right)\right)^{2} \exp \left(-\beta x_{r}^{-\alpha}\right)\left(-x_{r}^{-\alpha}\right)-\left(\exp \left(-\beta x_{r}^{-\alpha}\right)\right)^{2} 2\left(1-\exp \left(-\beta x_{r}^{-\alpha}\right)\right)\left(x_{r}^{\alpha}\right)\right] }
\end{aligned}
$$

$$
\begin{aligned}
\frac{\partial^{3} \ln L}{\partial \alpha^{2} \partial \beta}= & -\sum_{i=1}^{r} x_{i}^{-\alpha}\left(\ln x_{i}\right)^{2}-(n-r) x_{r}^{-\alpha}\left(\ln x_{r}\right)^{2} \\
& {\left[\frac{(1-A)\left(\beta x_{r}^{-\alpha}-A\right)}{A^{2}}+\frac{(1-A) A \beta x_{r}^{-\alpha}}{A^{2}}+\frac{\beta\left(\beta x_{r}^{-\alpha}-A\right)}{A^{4}} A(1-A)\left(x_{r}^{-\alpha}\right)(-A-2(1-A))\right], }
\end{aligned}
$$

$$
\begin{aligned}
\frac{\partial^{3} \ln L}{\partial \alpha \partial \beta^{2}}= & -(n-r)\left[\frac{-2(1-A) x_{r}^{-2 \alpha} \ln x_{r}}{A^{2}}+\frac{x_{r}^{-2 \alpha}}{\left(1-\exp \left(-\beta x_{r}^{-\alpha}\right)\right)^{4}}\left(\left(1-\exp \left(-\beta x_{r}^{-\alpha}\right)\right)^{2} \exp \left(-\beta x_{r}^{-\alpha}\right)\left(\beta x_{r}^{-\alpha} \ln x_{r}\right)\right.\right. \\
& \left.\left.+2\left(\exp \left(-\beta x_{r}^{-\alpha}\right)\right)^{2}\left(1-\exp \left(-\beta x_{r}^{-\alpha}\right)\right) \beta x_{r}^{-\alpha} \ln x_{r}\right)\right]
\end{aligned}
$$

\section{Data Availability}

This work is mainly a methodological development and has been applied on secondary data, but if required, data will be provided.

\section{Conflicts of Interest}

The authors declare that there are no conflicts of interest regarding the publication of this paper.

\section{References}

[1] E. Gumbel, Statistics of Extremes, Columbia University Press, New York, USA, 1958.

[2] K. Abbas and Y. Tang, "Estimation of parameters for Frechet distribution based on type-II censored samples," Caspian Journal of Applied Sciences Research, vol. 2, no. 7, pp. 36-43, 2013.

[3] H. M. Okasha, "E-Bayesian estimation for the Lomax distribution based on type-II censored data," Journal of the Egyptian Mathematical Society, vol. 22, no. 3, pp. 489-495, 2014. 
[4] H. H. Abu-Zinadah, "Bayesian estimation on the exponentiated Gompertz distribution under type II censoring," International Journal of Contemporary Mathematical Sciences, vol. 9, no. 11, pp. 497-505, 2014.

[5] R. M. El-Sagheer, "Bayesian prediction based on general progressive censored data from generalized Pareto distribution," Journal of Statistics Applications \& Probability, vol. 5, no. 1, pp. 43-51, 2016.

[6] K. Abbas, J. Fu, and Y. Tang, "Bayesian estimation of Gumbel type-II distribution," Data Science Journal, vol. 12, pp. 33-46, 2013.

[7] N. Feroze and M. Aslam, "Bayesian estimation of twocomponent mixture of gumbel type II distribution under informative priors," International Journal of Advanced Science and Technology, vol. 53, pp. 11-30, 2013.

[8] I. Malinowska and D. Szynal, "On characterization of certain distributions of $k^{\text {th }}$ lower (upper) record values," Applied Mathematics and Computation, vol. 202, no. 1, pp. 338-347, 2008.

[9] T. Sultana, M. Aslam, and M. Raftab, "Bayesian estimation of 3-component mixture of Gumbel type-II distributions under non-informative and informative priors," Journal of the National Science Foundation of Sri Lanka, vol. 45, no. 3, pp. 287-306, 2017.

[10] F. Metiri, H. Zeghdoudi, and M. R. Remita, "On Bayes estimates of Lindley distribution under Linux loss function: informative and non informative priors," Global Journal of Putre and Applied Mathematics, vol. 12, no. 1, pp. 391-400, 2016.

[11] H. Reyad and S. O. Ahmed, "E-Bayesian analysis of the Gumbel type-II distribution under type-II censored scheme," International Journal of Advanced Mathematical Sciences, vol. 3, no. 2, pp. 108-120, 2015.

[12] V. Preda, E. Panaitescu, and A. Constantinescu, "Bayes estimators of modified- weibull distribution parameters using Lindleys approximation," WSEAS Transactions on Mathematics, vol. 9, no. 7, pp. 539-549, 2010.

[13] A. Legendre, New Method for the Dermination of Orbits of Comets, Courcier, Paris, France, 1805.

[14] C. F. Gauss, Least Squares Method for the Combinations of Observation, (Translated by J. Bertrand 1955), MalletBachelier, Paris, France, 1810.

[15] H. R. Varian, A Bayesian Approach to Real Estate Assessment, pp. 195-208, North Holland, Amsterdam, Netherlands, 1975.

[16] R. Calabria and G. Pulcini, "Point estimation under asymmetric loss functions for left-truncated exponential samples," Communications in Statistics-Theory and Methods, vol. 25, no. 3, pp. 585-600, 1996.

[17] D. V. Lindley, “Approximate bayesian methods," Trabajos de Estadistica $Y$ de Investigacion Operativa, vol. 31, no. 1, pp. 223-245, 1980.

[18] E. T. Lee and J. W. Wang, Eds., Statistical Methods for Survival Data Analysis, John Wiley and Sons, New York, NY, USA, 3rd edition, 2003.

[19] S. W. Lagakos and J. S. Williams, "Models for censored survival analysis: a cone class of variable-sum models," Biometrika, vol. 65, pp. 181-189, 1978.

[20] S. Lee and R. A. Wolfe, "A simple test for independent censoring under the proportional hazards model," Biometrics, vol. 54, pp. 1176-1182, 1998. 